\title{
NON-DIVISIBILITY AMONG CHARACTER DEGREES II: NONSOLVABLE GROUPS
}

\author{
GUNTER MALLE AND ALEXANDER MORETÓ
}

\begin{abstract}
We say that a finite group $G$ is an NDAD-group (no divisibility among degrees) if for any $1<a<b$ in the set of degrees of the complex irreducible characters of $G, a$ does not divide $b$. In this article, we determine the nonsolvable NDAD-groups. Together with [13] this settles a problem raised by Berkovich and Zhmud', which asks for a classification of the NDAD-groups.
\end{abstract}

\section{IntRoduction AND STATEMENT OF RESUlts}

Let $G$ be a finite group. We say that $G$ is an NDAD-group (no divisibility among degrees) if for any $1<a<b$ in the set of degrees of the complex irreducible characters of $G, a$ does not divide $b$. Problem 58 of Berkovich and Zhmud' [1] asks for a classification of the NDAD-groups. Solvable NDADgroups where classified in [13]. In particular, it follows from the results there that a solvable NDAD-group has at most 4 character degrees. It was conjectured in [13] that there should exist a universal bound for the number of character degrees of an arbitrary NDAD-group. The goal of this paper is to classify the nonsolvable NDAD-groups, settling Berkovich and Zhmud's problem. The following is thus our main result.

Theorem A. Let $G$ be a nonsolvable NDAD-group. Write $C=C_{G}\left(G^{\prime}\right)$. Then $C=Z(G)$ and one of the following holds:

(i) $G=S \times C$, where $S$ is $J_{1}$ or ${ }^{2} B_{2}\left(2^{2 f+1}\right)$ for some $f>1$.

(ii) $G^{\prime}=\mathfrak{A}_{7}$ and $\left|G / C G^{\prime}\right| \leq 2$.

(iii) $G^{\prime}={ }^{2} B_{2}(8)$ and $\left|G / C G^{\prime}\right|$ divides 3 .

(iv) $G^{\prime}=\mathrm{L}_{3}(4)$ and $G / C=\mathrm{L}_{3}(4), \mathrm{L}_{3}(4) .2_{1}, \mathrm{~L}_{3}(4) .3$, or $\mathrm{L}_{3}(4) .6$.

(v) $G^{\prime}=\mathrm{L}_{2}(q)$ or $\mathrm{SL}_{2}(q)$ and $G / C=\operatorname{PGL}_{2}(q)$.

(vi) $G^{\prime}$ is $\mathfrak{A}_{6}$ or the 3 -fold cover of $\mathfrak{A}_{6}$ and $G / C=M_{10}$.

Conversely, if $C=Z(G)$ and any of (i)-(vi) holds, then $G$ is a nonsolvable NDAD-group.

As an immediate consequence of this theorem and the main result in [13], we can prove Conjecture A of [13].

Corollary B. Let $G$ be a finite NDAD-group. Then $|\operatorname{cd}(G)| \leq 7$.

As usual, given a group $G$ we write $\operatorname{cd}(G)$ to denote the set of degrees of the irreducible characters of $G$. B. Huppert [9] has conjectured that if $S$ is simple nonabelian and $G$ is a finite group such that $\operatorname{cd}(S)=\operatorname{cd}(G)$ then $G=S \times A$ for some abelian group $A$. The goal of [9] was to prove 
preliminary

this conjecture for the Suzuki simple groups and the fractional linear groups $\mathrm{L}_{2}\left(2^{f}\right)$ for any $f>1$. As a by-product of the classification of the NDADgroups, we obtain a new proof of Huppert's result (and a little bit more).

Corollary C (Huppert). If $S=\mathrm{L}_{2}\left(2^{f}\right)$ for some $f>1,{ }^{2} B_{2}\left(2^{2 f+1}\right)$ for some $f \geq 1, \mathrm{~L}_{3}(4), J_{1}$ or $\mathfrak{A}_{7}$ and $G$ is a finite group with $\operatorname{cd}(G)=\operatorname{cd}(S)$, then $G=S \times A$ for some abelian group $A$.

In Section 2 we classify the almost simple NDAD-group, using the classification of finite simple group. We prove in Section 3 that if $G$ is a nonsolvable NDAD-group, then $G^{\prime}$ is a quasi-simple group. Finally, we complete the proof of Theorem A in Section 4 and deduce corollaries B and C in Section 5 .

Some of this work was done while the second author was visiting the University of Kaiserslautern. He thanks the Mathematics Department there for its hospitality. The second author was partially supported by the FEDER, the Spanish Ministerio de Educación y Ciencia, the Generalitat Valenciana and Programa Ramón y Cajal.

\section{Almost simple Groups}

Recall that a finite group $G$ is almost simple if there exists a nonabelian simple group $S$ such that $S \leq G \leq \operatorname{Aut}(S)$. The goal of this section is to determine the almost simple NDAD-groups. We first discard the alternating groups:

Lemma 2.1. Let $G$ be an almost simple group with $F^{*}(G)=\mathfrak{A}_{n}, n \geq 8$. Then $G$ is not an NDAD-group.

Proof. Let's first consider the case where $G=\mathfrak{S}_{n}$ is the symmetric group. The irreducible characters of $\mathfrak{S}_{n}$ are labelled by partitions of $n$, and we write $\chi_{\lambda}$ for the character indexed by the partition $\lambda$. From the hook formula we obtain the following list of character degrees:

\begin{tabular}{r||c|c|c}
$\lambda$ & $\left(n-k, 1^{k}\right)$ & $(n-4,4)$ & $\left(n-4,2^{2}\right)$ \\
\hline$\chi_{\lambda}(1)$ & $\left(\begin{array}{l}n-1 \\
k-1\end{array}\right)$ & $\frac{n(n-1)(n-2)(n-7)}{24}$ & $\frac{n(n-1)(n-4)(n-5)}{12}$
\end{tabular}

If $n \not \equiv 3(\bmod 4)$ then $n-1$ divides $\frac{n(n-1)(n-4)(n-5)}{12}$. If $n \not \equiv 1(\bmod 3)$ then $n-1$ divides $\left(\begin{array}{c}n-1 \\ 3\end{array}\right)$. Finally, if $n \equiv 7(\bmod 12)$ then $\left(\begin{array}{c}n-1 \\ 2\end{array}\right)$ divides $n(n-1)(n-2)(n-7) / 24$. Note that the above partitions make sense for any $n \geq 8$. Moreover, they are different from the trivial partitions $(n)$ and $\left(1^{n}\right)$, so they label non-linear characters. Finally, for any $n \geq 8$ the two chosen degrees are different from each other. This shows the claim for symmetric groups.

Now note that $\chi_{\lambda}$ restricts irreducibly to $\mathfrak{A}_{n}$ unless $\lambda$ is a self-dual partition. Again, for $n \geq 8$ none of the partitions used above are self-dual, so we obtain the desired conclusion for $\mathfrak{A}_{n}$ as well.

For groups of Lie type we need some preparations. Let $\mathbf{G}$ be a semisimple algebraic group of adjoint type over the algebraic closure $\overline{\mathbb{F}}_{q}$ of a finite field $\mathbb{F}_{q}$, and let $F: \mathbf{G} \rightarrow \mathbf{G}$ be a Frobenius morphism on $\mathbf{G}$. Let $G:=\mathbf{G}^{F}$ denote the group of fixed points, a finite group of Lie type. Then any finite 
simple group of Lie type can be obtained as the derived group $G^{\prime}$ of $G$ for a suitable choice of $\mathbf{G}$ and $F$.

Let $W$ denote the Weyl group of $G$, that is, the group of fixed points under $F$ of the Weyl group of an $F$-stable maximal torus of $\mathbf{G}$ contained in an $F$-stable Borel subgroup. There is a natural bijection $\phi \mapsto \chi_{\phi}$ between $\operatorname{Irr}(W)$ and the set of constituents of the permutation character of $G$ on the Borel subgroup $B$ of $G$. The latter are called the unipotent principal series characters of $G$. Moreover the degree of $\chi_{\phi}$ is a polynomial in $q$ of the form

$$
\chi_{\phi}(1)=b_{a_{\phi}} q^{a_{\phi}}+\ldots+b_{A_{\phi}} q^{A_{\phi}}
$$

with increasing exponents $a_{\phi}<\ldots<A_{\phi}$ and rational coefficients $b_{i}$ such that $b_{a_{\phi}}, b_{A_{\phi}}$ are non-zero. Here, $a_{\phi}$, the order of vanishing at $q=0$, is the so-called $a$-invariant $a_{\phi}$ of $\chi_{\phi}$. (Note that this depends also on the Weyl group of $\mathbf{G}$, not just on $W$.)

Let $\epsilon \in \operatorname{Irr}(W)$ denote the sign-character of $W$. Then the unipotent principal series character $\chi_{\phi \otimes \epsilon}$ attached to $\phi \otimes \epsilon$ is the Alvis-Curtis dual of the unipotent character $\chi_{\phi}$ attached to $\phi$. It is known that the degrees of $\chi_{\phi}$ and its Alvis-Curtis dual only differ by a power of $q$.

Indeed, let $L: W \rightarrow \mathbb{N}_{0}$ denote the length function on $W$ and $\mathcal{H}=$ $\mathcal{H}(W, L)$ the corresponding Iwahori-Hecke algebra of $G$, a deformation of the group algebra of $W$. Then the degree of a principal series character $\chi_{\phi}$ is obtained from the Schur element $c_{\phi}$ of the Hecke algebra as $\chi_{\phi}(1)=$ $P / c_{\phi}$, where $P=\sum_{w \in W} q^{L(w)}$ denotes the Poincaré-polynomial of $G[5$, Cor. 9.3.6]. Let $S^{\prime}$ denote a set of representatives for the conjugacy classes of reflections in $W$ and, for $s \in S^{\prime}$, let $N_{s}$ be the number of occurrences of conjugates of $s$ in any shortest expression for the longest element $w_{0}$ of $W$.

Lemma 2.2. In the notation introduced above, let $\phi \in \operatorname{Irr}(W)$ denote an irreducible character of the Weyl group $W$ of $G, \tilde{\phi}=\phi \otimes \epsilon$ the tensor product with the sign character. Let $\chi, \tilde{\chi}$ be the corresponding unipotent principal series characters of $G$ with a-values a, $\tilde{a}$ respectively. Then

$$
\tilde{\chi}(1)=q^{\tilde{a}-a} \chi(1)
$$

where

$$
\tilde{a}-a=\sum_{s \in S^{\prime}} L(s) N_{s} \frac{\phi(s)}{\phi(1)} .
$$

Proof. By [5, Prop. 9.4.1(b) and 9.4.3] we have

$$
c_{\tilde{\phi}}=\operatorname{ind}\left(T_{w_{0}}\right) z_{\phi}^{-1} c_{\phi}
$$

with

$$
z_{\phi}=\prod_{s \in S^{\prime}} u_{s}^{N_{s}(1+\phi(s) / \phi(1))}
$$

by [5, Th. 9.2.2]. Here $u_{s}=q^{L(s)}$ are the parameters of the Hecke algebra $\mathcal{H}$ and

$$
\operatorname{ind}\left(T_{w_{0}}\right)=q^{L\left(w_{0}\right)}=q^{\sum_{s \in S^{\prime}} L(s) N_{s}} .
$$

Thus

$$
\tilde{\chi}(1)=\frac{P}{c_{\tilde{\phi}}}=\frac{P}{c_{\phi}} \frac{z_{\phi}}{\operatorname{ind}\left(T_{w_{0}}\right)}=\chi(1) \prod_{s \in S^{\prime}} q^{L(s) N_{s} \phi(s) / \phi(1)}
$$


as claimed.

In particular, for any $\phi \in \operatorname{Irr}(W)$, one of the degrees $\chi_{\phi}(1), \chi_{\phi \otimes \epsilon}(1)$ is a multiple of the other. In order to obtain character degrees which are proper multiples of each other it hence suffices to ensure that $a_{\phi \otimes \epsilon}-a_{\phi} \neq 0$ for suitable $\phi \in \operatorname{Irr}(W)$. The most natural candidate, which moreover exists for any Weyl group, is certainly the reflection character.

Lemma 2.3. In the notation introduced above, let $\rho \in \operatorname{Irr}(W)$ denote the reflection character of the Weyl group $W$ of $G, \tilde{\rho}=\rho \otimes \epsilon$ the tensor product with the sign character. Let $\chi, \tilde{\chi}$ be the corresponding unipotent principal series character of $G$. Then $1<\chi(1)<\tilde{\chi}(1)$ and $\chi(1)$ divides $\tilde{\chi}(1)$, unless $W$ has rank 1 or 2.

Proof. Clearly, the value of $\rho$ on reflections equals $\rho(1)-2$, so we find that

$$
a_{\tilde{\rho}}-a_{\rho}=\frac{\rho(1)-2}{\rho(1)} \sum_{s \in S^{\prime}} L(s) N_{s}
$$

by Lemma 2.2. This is positive if and only if $\rho(1)>2$, that is, for $W$ of rank at least 3 .

The degree $\chi(1)$, interpreted as a polynomial in $q$, is a product of cyclotomic polynomials in $q$. At $q=1$ it specializes to the degree $\rho(1) \geq 1$ of $\rho$. In particular, it is not divisible by the linear polynomial $(q-1)$. It is easy to see that any other cyclotomic polynomial is strictly increasing on $[1, \infty]$ (check the real factors of degree 2), thus $\chi(1)$, the value at $q$, is larger than $\rho(1)$, hence larger than 1 .

We next show that the particular unipotent characters considered above extend to the full automorphism group. For this let $\hat{\mathbf{G}}$ be a simply connected covering group of $\mathbf{G}$, that is, $\hat{\mathbf{G}}$ is simply connected and has a morphism $\hat{\mathbf{G}} \rightarrow \mathbf{G}$ with central kernel. Let $\hat{F}: \hat{\mathbf{G}} \rightarrow \hat{\mathbf{G}}$ denote a lift of $F$ to $\hat{\mathbf{G}}$, with fixed point group $\hat{G}=\hat{\mathbf{G}}^{\hat{F}}$. Then we obtain an induced homomorphism $\hat{G} \rightarrow$ $G$. Let's write $G^{\prime}$ for the image of this homomorphism; it coincides with the derived subgroup of $G$ unless $G^{\prime}$ is not simple. According to Lusztig's theory, the unipotent characters of $\hat{G}$ have $Z(\hat{G})$ in their kernel. Thus they may be considered as characters of $G^{\prime}$, and they coincide with the restrictions to $G^{\prime}$ of the unipotent characters of $G$. In particular, the unipotent characters of $G$ restrict irreducibly to $G^{\prime}$.

Proposition 2.4. Let $G, G^{\prime}$ be as above, $\chi$ the unipotent principal series character of $G$ indexed by the reflection character of the Weyl group $W$ of $G$ and $\tilde{\chi}$ its Alvis-Curtis dual. Then the (irreducible) restrictions to $G^{\prime}$ of both $\chi$ and $\tilde{\chi}$ extend to $\operatorname{Aut}\left(G^{\prime}\right)$.

Proof. By a result of Steinberg Out $\left(G^{\prime}\right)$ is a solvable group generated by the diagonal automorphisms, the graph automorphisms and the field automorphisms of $G^{\prime}$ (see [6, Th. 2.5.1]). The group $G$ is just the subgroup of Aut $\left(G^{\prime}\right)$ generated by $G^{\prime}$ together with the group of diagonal automorphisms. We hence have to check that $\chi, \tilde{\chi}$ are invariant under graph and field automorphisms and extend to $\operatorname{Aut}\left(G^{\prime}\right)$. Now by construction the unipotent characters are invariant under all field automorphisms. The graph automorphisms are induced by graph automorphisms of the Weyl group $W$ which in 
turn by definition fix the reflection representation. Thus the unipotent character corresponding to the reflection character of $W$ is also invariant under all graph automorphisms. The same reasoning applies to the sign character of $W$, and hence to $\tilde{\chi}$. It follows that $\chi, \tilde{\chi}$ are invariant in $\operatorname{Aut}\left(G^{\prime}\right)$.

The group of field automorphisms is cyclic, thus we are done unless $G$ has non-trivial graph automorphisms. Assume that $\Gamma$ is an $F$-invariant group of graph automorphisms of $\mathbf{G}$. Then according to Digne-Michel [4] there exist generalized Deligne-Lusztig characters for the group of fixed points $(\mathbf{G} . \Gamma)^{F}$ of the disconnected group G.Г. Unipotent characters can be defined in the same way as in the connected case, and these are again invariant under all field automorphisms. Moreover, their restrictions to $G$ are just the sums over $\Gamma$-orbits of unipotent characters of $G$. In particular, if $\chi$ is a $\Gamma$-invariant unipotent character of $G$, it extends to a unipotent character of $(\mathbf{G} \cdot \Gamma)^{F}$ which is still invariant under field automorphisms. The latter extend further to the whole of $\operatorname{Aut}\left(G^{\prime}\right)$ and we are done.

To treat $\mathrm{L}_{2}(q)$ we need the following easy observation:

Lemma 2.5. Let $q=p^{f} \geq 16, f>1$, be a prime power.

(a) There exists $s \in \mathbb{F}_{q}^{\times}$such that $s^{2}+s^{-2}$ is a primitive element of $\mathbb{F}_{q}$.

(b) There exists $s \in \mathbb{F}_{q^{2}}^{\times}$of norm 1 over $\mathbb{F}_{q}$ such that $s^{2}+s^{-2}$ is a primitive element of $\mathbb{F}_{q^{2}}$.

Proof. For (a) we estimate the number of elements of $\mathbb{F}_{q}$ lying in proper subfields. The subfields of $\mathbb{F}_{q}$ are the fields $\mathbb{F}_{p^{a}}$ for divisors $a \mid f$. Hence the number $m$ of non-primitive elements of $\mathbb{F}_{q}$ can be estimated by

$$
m \leq \sum_{a \mid f, a<f} p^{a} \leq \sum_{a=1}^{f / 2} p^{a} \leq 2 p^{f / 2} .
$$

The map $\mathbb{F}_{q}^{\times} \rightarrow \mathbb{F}_{q}^{\times}, s \mapsto s^{2}+s^{-2}$, has fibers of size at most 4 , and at most 2 if $q$ is even. So at least $q / 4$ elements of $\mathbb{F}_{q}$ are of the form $s^{2}+s^{-2}$, and at least $q / 2$ if $q$ is even. Hence there exist primitive elements of the form $s^{2}+s^{-2}$ if $2 \sqrt{q}<q / 4$, that is, if $q \geq 65$, and also for $q \geq 17$ if $q$ is even. If $q=p^{2}$, so $f=2, q-\sqrt{q}$ elements are primitive, so we are done when $q \geq 25$. In $\mathbb{F}_{27}$ only three elements are not primitive, which is less than $27 / 4$. Explicit calculation shows that the statement holds when $q=16$.

For (b) we may argue similarly, this time counting norm 1 elements lying in proper subfields of $\mathbb{F}_{q^{2}}$. The analogous estimate as above shows that there are at most $2 p^{f / 3}$ such elements. With this, we are done when $q \geq 22$. For $q=16$, the claim follows by direct calculation.

Lemma 2.6. Let $G$ be an almost simple group with $F^{*}(G)=\mathrm{L}_{2}(q), q=$ $p^{f}>4$. If $G$ is an NDAD-group then $G=\mathrm{PGL}_{2}(q)$ or $G=M_{10}$.

Proof. We may assume that $q \geq 11$, since the other cases can be checked from the Atlas (which leads in particular to the case $M_{10}=\mathfrak{A}_{6} \cdot 2_{3}$ ). Let $S=F^{*}(G)=\mathrm{L}_{2}(q)$. First assume that $q=p^{f} \geq 11$ is odd, $q \equiv \epsilon(\bmod 4)$ with $\epsilon \in\{ \pm 1\}$. Then the irreducible characters of $S$ have degrees $1, q$ (one each) $q-1((q-1) / 2$ many), $q+1((q-3) / 2$ many) and $(q+\epsilon) / 2$ (two). Thus $S$ itself does not satisfy NDAD. The outer automorphism group of $S$ is 
the direct product of the group of diagonal automorphisms, of order 2, and the group of field automorphisms of order $f$. The diagonal automorphism of order two fuses the two characters of degree $(q+\epsilon) / 2$ and leaves all other characters invariant. Thus the extension $\mathrm{PGL}_{2}(q)$ of $S$ with the diagonal automorphism is an NDAD-group.

We may hence assume that $G$ is such that $H:=G /\left(G \cap \mathrm{PGL}_{2}(q)\right)$ is nontrivial (and necessarily cyclic). In particular $q \neq p$. Let $\sigma$ denote a generator of $H$; it acts like a non-trivial field automorphism $\gamma$ on the characters of $S$ of degrees $q \pm 1$. Let $M^{ \pm}$denote the set of characters of degree $q \pm 1$ of $S$. Then $\left|M^{-}\right|=\left|M^{+}\right|+1$. So, if $\gamma$ acts faithfully on both $M^{-}, M^{+}$, then on at least one of the two sets there must occur a non-regular orbit as well. But then we obtain two different character degrees, one dividing the other. It is sufficient to prove faithfulness in the case that $\gamma$ is a generator of the group of field automorphisms. The irreducible characters in $M^{+}$are parametrized by elements of $\mathbb{F}_{q}^{\times}$of the form $t=s^{2}+s^{-2}, s^{4} \neq 1$, and $\gamma$ acts by $t \mapsto t^{p}$. Thus $\gamma$ has a regular orbit if there exists a primitive element of $\mathbb{F}_{q}$ of the form $s^{2}+s^{-2}$. By Lemma 2.5(a), such elements exist for $11 \leq q \neq p$. Similarly, irreducible characters in $M^{-}$are parametrized by elements $t=s^{2}+s^{-2}$, where $s \in \mathbb{F}_{q^{2}}^{\times}$has norm 1 and $s^{4} \neq 1$. Again, regular $\gamma$-orbits exist by Lemma $2.5(\mathrm{~b})$.

Now let $q \geq 16$ be even. In this case, $S$ has irreducible characters of degree $1, q$ (one each), $q-1(q / 2$ many) and $q+1((q-2) / 2$ many). In particular $S$ is an NDAD-group. The only outer automorphisms are field automorphisms, and with Lemma 2.5 we may conclude that all $G \neq S$ with $F^{*}(G)=S$ are not NDAD.

Lemma 2.7. Let $G$ be an almost simple group with $F^{*}(G)={ }^{2} B_{2}\left(2^{2 f+1}\right)$, $f \geq 1$. If $G$ is an NDAD-group then $G={ }^{2} B_{2}\left(2^{2 f+1}\right)$ or $G={ }^{2} B_{2}(8) .3$

Proof. The case $f=1$ can be checked from the Atlas so assume that $f \geq 2$. The group $S={ }^{2} B_{2}\left(q^{2}\right), q^{2}=2^{2 f+1}$, has unipotent characters of degrees $1, \sqrt{2} q\left(q^{2}-1\right) / 2(2 \times)$ and $q^{4}$, and further irreducible characters of degree $q^{4}+1\left(\left(q^{2}-2\right) / 2\right.$ many $),\left(q^{2}-1\right)\left(q^{2} \pm \sqrt{2} q+1\right)\left(\left(q^{2} \mp \sqrt{2} q\right) / 4\right.$ many $)$. Thus $S$ is NDAD. The outer automorphism group of $S$ is cyclic of order $2 f+1$ and consists of field automorphisms. So now assume that $G / S$ is nontrivial, generated by a field automorphism $\sigma$. The characters of degree $\left(q^{2}-1\right)\left(q^{2} \mp \sqrt{2} q+1\right)$ are indexed by elements of the cyclic maximal torus $T^{ \pm}$of order $\left(q^{2} \pm \sqrt{2} q+1\right)$ modulo conjugation in its normalizer $N_{S}\left(T^{ \pm}\right)$in a way compatible with the action of field automorphisms. The normalizer quotient $N_{S}\left(T^{ \pm}\right) / T^{ \pm}$is cyclic and acts semiregularly on the nonidentity elements of $T^{ \pm}$. Thus to show that $G$ does not satisfy NDAD, it suffices to produce two $N\left(T^{ \pm}\right)$-orbits of non-identity elements of $T^{ \pm}$with different orbit length under $\sigma$. Note that $q^{4}+1=2^{4 f+2}+1 \equiv 0(\bmod 5)$, so one of $q^{2} \pm \sqrt{2} q+1$ is divisible by 5 . We let $T$ denote the maximal torus whose order is divisible by 5 . All elements of order 5 in $T$ are conjugate in $N_{S}(T)$, so this $N_{S}(T)$-class of $T$ is stable under all field automorphisms, and the same holds for the corresponding irreducible character. On the other hand, if we take a generator $s$ of $T$, then its $N_{S}(T)$-class is not stable under any 
nontrivial field automorphism (since $q^{2} \geq 32$ ). Thus the same holds for the associated character, and we have shown that $G$ is not an NDAD-group.

We are now ready to classify the almost simple NDAD-groups:

Theorem 2.8. Let $G$ be an almost simple NDAD-group. Then $G$ is one of the following groups: $\mathrm{PGL}_{2}(q), M_{10}, \mathrm{~L}_{3}(4), \mathrm{L}_{3}(4) .2_{1}, \mathrm{~L}_{3}(4) .3, \mathrm{~L}_{3}(4) .6$, ${ }^{2} B_{2}\left(2^{2 f+1}\right),{ }^{2} B_{2}(8) .3, \mathfrak{A}_{7}, \mathfrak{S}_{7}$ or $J_{1}$.

Proof. Let $G$ be almost simple, that is, the generalized Fitting subgroup $S=F^{*}(G)$ is a finite simple group. By the classification $S$ is an alternating group, a group of Lie type or a sporadic group. The 26 sporadic groups can be easily checked from the Atlas [3], leading to the single case of $J_{1}$.

If $S$ is an alternating group $\mathfrak{A}_{n}$, it is not NDAD for $n \geq 8$ by Lemma 2.1. The groups $\mathfrak{A}_{5}$ and $\mathfrak{A}_{6}$ are isomorphic to the Lie type groups $\mathrm{L}_{2}(5), \mathrm{L}_{2}(9)$ respectively, and $\mathfrak{A}_{7}, \mathfrak{S}_{7}$ are exceptions in the statement.

So now assume that $S$ is of Lie type. Then $S$ may be obtained as a group $G^{\prime}$ from the group of fixed points $G$ of a simple algebraic group of adjoint type $\mathbf{G}$ as explained above, unless $S={ }^{2} F_{4}(2)^{\prime}$. Again the latter group and its automorphism group can be checked from [3]. Otherwise, first assume that $G$ is of rank at least 3. Then by Lemma 2.3 the group $G$ is not NDAD, and by Proposition 2.4 the same is true for all subgroups $S \leq K \leq \operatorname{Aut}(S)$.

If $G$ has rank at most 2, then it is of one of the following types:

$$
A_{1}, A_{2},{ }^{2} A_{2},{ }^{2} A_{3},{ }^{2} A_{4}, B_{2},{ }^{2} B_{2},{ }^{3} D_{4},{ }^{2} F_{4}, G_{2},{ }^{2} G_{2} \text {. }
$$

For $G$ of type ${ }^{2} A_{3}$ or ${ }^{2} A_{4}$ we may take the Ennola-duals of the unipotent characters that we chose for $A_{3}, A_{4}$ respectively, and these will do the job. For type ${ }^{2} F_{4}$ we take the unipotent characters labelled by $\varepsilon^{\prime}, \varepsilon^{\prime \prime}$ in [2, p.489], for type ${ }^{3} D_{4}$ we take those labelled by $\phi_{1,3}^{\prime}, \phi_{1,3}^{\prime \prime}$ in $[2$, p.478]. In all these cases, the chosen characters extend to $\operatorname{Aut}\left(G^{\prime}\right)$ by the same arguments as those given in Proposition 2.4.

For the remaining types, there are no examples among unipotent characters, so we have to choose characters in other Lusztig series. For type ${ }^{2} G_{2}$ we take the two characters of degree $q^{4}-q^{2}+1, q^{2}\left(q^{4}-q^{2}+1\right)$ lying in the Lusztig series of an involution in the dual group [17].

For $G$ of type $G_{2}$ take the characters of degrees $q^{4}+q^{2}+1, q^{2}\left(q^{4}+q^{2}+1\right)$ corresponding to an involution in the dual group when $q$ is odd, and the characters of degrees $q^{3}+\epsilon, q^{3}\left(q^{3}+\epsilon\right)$ corresponding to an element of order 3 in the dual group when $q \equiv \epsilon(\bmod 3)$ is even.

For $G$ of type $B_{2}$, the four dimensional symplectic group, we take the characters of degrees $\left(q^{2}+1\right) / 2$ and $q^{2}\left(q^{2}+1\right) / 2$ corresponding to an involution in the dual group with centralizer of type $A_{1} \times A_{1}$ when $q$ is odd (the first is a Weil character of $G$ ), and characters of degrees $(q-1)\left(q^{2}+1\right)$ and $q(q-1)\left(q^{2}+1\right)$ corresponding to an element of order $q+1$ with centralizer of type $A_{1}(q)$ if $q$ is even. For all three types ${ }^{2} G_{2}, G_{2}$ and $B_{2}$, the outer automorphism group is cyclic, and the characters we chose are invariant under automorphisms, so they extend to the full automorphism group.

The dual group of $G=\operatorname{PGL}_{3}(q)$ is $G^{*}=\mathrm{SL}_{3}(q)$. Let $s \in G^{*}$ be an element of order $q-1$ with centralizer $\mathrm{GL}_{2}(q)$. (Such elements exists if $(q-1) / \operatorname{gcd}(3, q-1) \neq 1$, so for $q \neq 2,4$. But $\mathrm{PGL}_{3}(2) \cong \mathrm{L}_{2}(7)$ was 
already considered, and $\mathrm{PGL}_{3}(4)$ occurs in the statement.) Then the Lusztig series in $\operatorname{Irr}(G)$ corresponding to $s$ contains irreducible characters of degrees $q^{2}+q+1$ and $q\left(q^{2}+q+1\right)$. Both characters behave the same under field automorphisms. The graph automorphism has order 2 ; since $G$ also has characters of degrees $(q-1)\left(q^{2}+q+1\right)$ and $(q+1)\left(q^{2}+q+1\right)$, extensions with the graph automorphism are also not NDAD-groups.

For $G=\mathrm{PGU}_{3}(q)$ we choose an element $s$ in the dual group $G^{*}=\operatorname{SU}_{3}(q)$ of order $q+1$ and with centralizer $\mathrm{GU}_{2}(q)$. Such an element exists unless $(q+1) / \operatorname{gcd}(3, q+1)=1$, so unless $q=2$. But $\mathrm{PGU}_{3}(2)$ is solvable. Here, the characters in the corresponding Lusztig series have degrees $q^{2}-q+1$ and $q\left(q^{2}-q+1\right)$. The behaviour under automorphisms is the same as in the previously treated case of $\mathrm{PGL}_{3}(q)$.

Finally, for $S={ }^{2} B_{2}\left(2^{2 f+1}\right)$ see Lemma 2.7, for $S=\mathrm{L}_{2}(q)$ see Lemma 2.6.

\section{THE REDUCTION}

In this section, we prove that if $G$ is a nonsolvable NDAD-group, then $G^{\prime}$ is a quasi-simple group, i.e., that it is perfect and $G^{\prime} / Z\left(G^{\prime}\right)$ is simple nonabelian. We begin by proving that $G^{\prime}$ is perfect.

Lemma 3.1. Let $G$ be a nonsolvable NDAD-group. Then $G^{\prime}$ is perfect.

Proof. Assume not. Let $U$ be the smallest normal subgroup of $G$ such that $G / U$ is solvable. Take a chief factor $U / V$, which is a direct product of copies of a nonabelian simple group $S$. We know that there exists $\alpha \in \operatorname{Irr}(S)$ nonprincipal that extends to $\operatorname{Aut}(S)$ (by Lemma 4.2 of [15], for instance). Hence $\alpha \times \cdots \times \alpha \in \operatorname{Irr}(U / V)$ extends to $\tau \in \operatorname{Irr}(G)$. If we take a nonlinear character $\gamma \in \operatorname{Irr}(G / U)$ we have that $\gamma \tau \in \operatorname{Irr}(G)$ and $\tau(1)$ properly divides $\gamma \tau(1)$. This contradiction proves the lemma.

Our next goal is to prove that any composition series of our nonsolvable NDAD-group has a unique nonabelian composition factor. This is accomplished in the next two lemmas.

Lemma 3.2. Let $U / V$ be a nonabelian chief factor of an NDAD-group $G$. Then $U / V$ is simple.

Proof. As before, $U / V$ is a direct product of copies of a nonabelian simple group $S$, and we can take $\alpha \in \operatorname{Irr}(S)$ that extends to $\operatorname{Aut}(S)$. Assume that $U / V$ is not simple. Take $\beta \in \operatorname{Irr}(S)-\left\{1_{S}, \alpha\right\}$. Put $\varphi=\alpha \times 1_{S} \times \cdots \times 1_{S}$ and $\psi=\alpha \times \beta \times \cdots \times \beta$. We have that $T=I_{G}(\varphi) \geq I_{G}(\psi)$. Furthermore, $\varphi(1)$ properly divides $\psi(1)$ and $\varphi$ extends to $\nu \in \operatorname{Irr}(T)$. Then $\nu^{G} \in \operatorname{Irr}(G)$ and $\nu^{G}(1)$ properly divides the degree of any irreducible constituent of $\psi^{G}$. This is a contradiction.

Lemma 3.3. Let $G$ be a nonsolvable NDAD-group. Then any composition series has a unique nonabelian composition factor.

Proof. Argue as in the proof of Lemma 3.1: if $G / U$ is not solvable and $U / V$ is simple, then we can find some irreducible character of $U / V$ that extends to $\tau \in \operatorname{Irr}(G)$. But now, for any nonlinear $\psi \in \operatorname{Irr}(G / U), \chi=\tau \psi \in \operatorname{Irr}(G)$ and $\tau(1)$ properly divides $\chi(1)$. 
Now we have the following.

Corollary 3.4. Let $G$ be a nonsolvable NDAD-group. Let $G^{\prime} / U$ be a chief factor. Then $G^{\prime} / U=\mathrm{L}_{3}(4), J_{1}, \mathfrak{A}_{7},{ }^{2} B\left(2^{2 f+1}\right)$ for some $f \geq 1$ or $\mathrm{L}_{2}(q)$ for some $q \geq 4$ and $U$ is solvable.

Proof. By Lemma 3.1 and Lemma 3.2, $G^{\prime} / U$ is a nonabelian simple group. Now, Lemma 3.3 implies that $U$ is solvable. Write $C / U=C_{G / U}\left(G^{\prime} / U\right)$. Then $G / C$ is an almost simple NDAD-group and $G^{\prime} C / C \cong G^{\prime} / U$. Now the list of possibilities for $G^{\prime} / U$ follows from Theorem 2.8.

In the remainder of this section we will use the notation of Corollary 3.4. In particular, $G^{\prime} / U$ will be a chief factor and $C / U=C_{G / U}\left(G^{\prime} / U\right)$. Recall also that $G / C$ is one of the groups in the statement of Theorem 2.8. Our next goal is to prove that any character of $U$ is $G^{\prime}$-invariant. We will argue case by case according to the different possibilities for $G^{\prime} / U$ in Corollary 3.4 .

Lemma 3.5. Let $G$ be a nonsolvable $N D A D$-group and assume that $G^{\prime} / U=$ $\mathrm{L}_{3}(4)$. Then every irreducible character of $U$ is $G^{\prime}$ - invariant.

Proof. Assume that there is some irreducible character $\lambda$ of $U$ that is not $G^{\prime}$-invariant and let $T<G^{\prime}$ be the inertia group of $\lambda$ in $G^{\prime}$. We want to see that in this case $G$ is not an NDAD-group.

By Theorem $2.8 G / C$ is $\mathrm{L}_{3}(4), \mathrm{L}_{3}(4) .2_{1}, \mathrm{~L}_{3}(4) .3$, or $\mathrm{L}_{3}(4) .6$. We claim that in this case there is some irreducible character of $G^{\prime}$ whose degree is a proper multiple of some irreducible character degree of $G / C$. This will conclude the proof. Since when ordered by divisibility the members of $\operatorname{cd}\left(\mathrm{L}_{3}(4) .6\right)$ are maximal members among the character degrees of our four possibilities for $G / C$, we may assume that $G / C=\mathrm{L}_{3}(4) .6$. The set of character degrees of this group is

$$
\operatorname{cd}\left(\mathrm{L}_{3}(4) .6\right)=\{1,20,105,90,126,64\} .
$$

Using the classification of the maximal subgroups of $\mathrm{L}_{3}(4)$ in [3] we see that the maximal subgroups of $G^{\prime}$ that contain $U$ have indices $280,120,56$ and 21. If $T$ is contained in a maximal subgroup of index 280 or 120 , it is clear that any irreducible character of $G^{\prime}$ lying over $\lambda$ will be a proper multiple of 20. This proves the claim in this case. Hence we may assume that $T$ is contained in a maximal subgroup of $G^{\prime}$ of index 56 or 21 .

The subgroups of index 56 of $G^{\prime} / U$ are isomorphic to $\mathrm{L}_{2}(9)$. Assume first that $T / U$ is one of these subgroups. We are interested in the degrees of the characters of $T$ lying over $\lambda$. Using a character isomorphism triple (see Chapter 11 of [11]), we may assume that $U$ is central in $T$. Looking at the character degrees of the central extensions of $\mathrm{L}_{2}(9)$ in the Atlas, we see that $T$ has some character of degree either 8 or 9 lying over $\lambda$. Since this character induces irreducibly to $G^{\prime}$ we conclude that $G^{\prime}$ has some character of degree either $56 \cdot 8 \cdot \lambda(1)$ (which is a proper multiple of 64 ) or $56 \cdot 9 \cdot \lambda(1$ ) (which is a proper multiple of 126). Hence we may assume that $T$ is a proper subgroup of a maximal subgroup of $G^{\prime}$ of index 56. The indices of the maximal subgroups of $\mathrm{L}_{2}(9)$ are 15,10 and 6 . Since $56 \cdot 15$ and $56 \cdot 10$ are proper multiples of 20 , we may assume that $T$ is contained in some subgroup 
of index $56 \cdot 6=2^{4} \cdot 3 \cdot 7$ of $G^{\prime}$. The subgroups of $G^{\prime} / U$ of this index that are contained in some subgroup isomorphic to $L_{2}(9)$ are isomorphic to $\mathfrak{A}_{5}$. Assume for the moment that $T / U$ is isomorphic to $\mathfrak{A}_{5}$. Using a character isomorphism triple again we may assume that $U$ is central in $T$ and looking at the character degrees of the central extensions of $\mathfrak{A}_{5}$ in the Atlas we deduce that $T$ has some character of degree $4 \lambda(1)$ lying over $\lambda$. Hence $G^{\prime}$ has some character of degree $2^{6} \cdot 3 \cdot 7 \cdot \lambda(1)$ lying over $\lambda$. Since this is a multiple of 64 , the claim follows in this case. Finally, we may assume that $T / U$ is a proper subgroup of our second maximal subgroup of $G^{\prime} / U$ isomorphic to $\mathfrak{A}_{5}$. Since either 3 or 5 divides the index of any proper subgroup of $\mathfrak{A}_{5}$, we deduce in any case that there is some character of $G^{\prime}$ lying over $\lambda$ that is a proper multiple of some character degree of $G / C$.

Finally, we may assume that $T$ is contained in some subgroup of $\mathrm{L}_{3}(4)$ of index 21. The subgroups of $\mathrm{L}_{3}(4)$ of this index are isomorphic to the perfect group $2^{4}: \mathfrak{A}_{5}$. Assume first that $T / U=2^{4}: \mathfrak{A}_{5}$. As before, we may assume that $U$ is cyclic and central in $T$ and that $\lambda$ is faithful. Write $N$ to denote the normal subgroup of $T$ such that $N / U=2^{4}$. Clearly, $N$ is nilpotent. If $N$ is not abelian, then $2 \lambda(1)$ divides the degree of any character $\psi$ of $N$ lying over $\lambda$. If $\psi$ is $T$-invariant then, again as before, we see that $5 \psi(1)$ or $6 \psi(1)$ divides the degree of some character of $T$ lying over $\psi$. Hence $2 \cdot 5 \cdot 21$ or $2 \cdot 6 \cdot 21$ divides the degree of some character of $G^{\prime}$ lying over $\lambda$. Since these numbers are proper multiples of 105 and 126 respectively, we conclude the result in this case. If $\psi$ is not $T$-invariant either 5 or 6 divides the index in $T$ of the inertia group of $\psi$. We deduce that again either $2 \cdot 5 \cdot 21$ or $2 \cdot 6 \cdot 21$ divides the degree of some irreducible character of $G^{\prime}$ lying over $\lambda$. Hence, we may assume that $N$ is abelian. If $N$ can be written as a direct product of $U$ and another subgroup $V \cong 2^{4}$, then we can take the character $\lambda \times \nu$, for any nonprincipal character $\nu$ of $V$. Since we know that $\mathfrak{A}_{5}$ acts transitively on the nonprincipal characters of $V$, we deduce that $\lambda \times \nu$ lies in a $T$-orbit of size 15 . We conclude that $15 \cdot 21$ divides the degree of some irreducible character of $G^{\prime}$ lying over $\lambda$ and this is a proper multiple of 105 . Thus we may assume that the abelian group $N$ cannot be written as a direct product of $U$ and another subgroup. In particular, 4 divides the exponent of $N$ and the order of any character $\tau$ of $N$ lying over $\lambda$ is a multiple of 4 . We also have that the characters of $N$ of order 2 are exactly the nonprincipal characters of $N$ whose kernel contains $U$. Recall that $T$ acts transitively on these characters. Also, since $\tau^{o(\tau) / 2}$ has order 2, we deduce that the size of the $T$-orbit of $\tau$ is a multiple of 15 . Again, we conclude that $15 \cdot 21$ divides the degree of some irreducible character of $G^{\prime}$ lying over $\lambda$.

Now, we may assume that $T / U$ is a proper subgroup of $2^{4}: \mathfrak{A}_{5}$. The maximal subgroups of this latter group have indices $16,10,6$ and 5 . If $T$ is contained in some of the subgroups whose index is 10,6 , or 5 , it is easy to find some character of $G^{\prime}$ lying over $\lambda$ whose degree is a proper multiple of 105 or 126 . Hence, we may assume that $T$ is contained in one of the subgroups of index 16 . Notice that these subgroups are isomorphic to $\mathfrak{A}_{5}$ and have already been excluded before.

Lemma 3.6. Let $G$ be a nonsolvable $N D A D$-group and assume that $G^{\prime} / U=$ $J_{1}$. Then every irreducible character of $U$ is $G^{\prime}$-invariant. 
Proof. In this case, one can easily check in the Atlas that the index of every maximal subgroup of $G^{\prime} / U=J_{1}$ is a proper multiple of the degree of some irreducible character of $J_{1}$. Since the character degrees of $J_{1}$ are character degrees of $G$ (because $\operatorname{Out}\left(J_{1}\right)=1$ ), we deduce that all the irreducible characters of $U$ are $G^{\prime}$-invariant, as desired.

Lemma 3.7. Let $G$ be a nonsolvable $N D A D$-group and assume that $G^{\prime} / U=$ $\mathfrak{A}_{7}$. Then every irreducible character of $U$ is $G^{\prime}$ - invariant.

Proof. Now, we have $G / C=\mathfrak{A}_{7}$ or $\mathfrak{S}_{7}$. Assume that there is some irreducible character $\lambda$ of $U$ that is not $G^{\prime}$-invariant and let $T<G^{\prime}$ be the inertia group of $\lambda$ in $G^{\prime}$. We want to see that in this case $G$ is not an NDAD-group. We claim that in this case there is some irreducible character of $G^{\prime}$ whose degree is a proper multiple of some irreducible character degree of $G / C$. This will conclude the proof.

As in the case when $G^{\prime} / U$ was $\mathrm{L}_{3}(4)$, we may assume that

$$
\operatorname{cd}(G / C)=\operatorname{cd}\left(\mathfrak{S}_{7}\right)=\{1,14,15,20,21,35\} .
$$

The maximal subgroups of $\mathfrak{A}_{7}$ have indices 7, 15, 21 and 35. The indices $15,21,35$ coincide with character degrees of $G / C$ and the subgroups of these indices are not abelian. The claim follows in this case from Gallagher's Theorem (Corollary 6.17 of [11]) and Clifford's Theorem (Theorem 6.11 of [11]). Thus, we may assume that $T$ is contained in a subgroup of index 7 of $\mathfrak{A}_{7}$. These subgroups are isomorphic to $\mathfrak{A}_{6}$. If $T=\mathfrak{A}_{6}$ then we can find arguing as before characters of $T$ lying over $\lambda$ whose degree is a multiple of 6 or 8 . This yields characters of $G^{\prime}$ lying over $\lambda$ whose degree is a multiple of $6 \cdot 7$ or $8 \cdot 7$ and both of these numbers are proper multiples of 14 .

If $T$ is proper in $\mathfrak{A}_{6}$ it suffices to note that the indices of the maximal subgroups of $\mathfrak{A}_{6}$ are 6,10 and 15 .

Lemma 3.8. Let $G$ be a nonsolvable $N D A D$-group and assume that $G^{\prime} / U=$ ${ }^{2} B_{2}\left(q^{2}\right)$, where $q^{2}=2^{2 f+1}$ for some $f \geq 1$. Then every irreducible character of $U$ is $G^{\prime}$-invariant.

Proof. As in the previous lemma, assume that there is some irreducible character $\lambda$ of $U$ that is not $G^{\prime}$-invariant and let $T<G^{\prime}$ be the inertia group of $\lambda$ in $G^{\prime}$.

First, we assume that $G / C={ }^{2} B_{2}\left(q^{2}\right)=G^{\prime} / U$. Then, by [10, Theorem XI.5.10] and the Atlas,

$\operatorname{cd}(G / C)=\left\{1, q^{4}, q^{4}+1,\left(q^{2}-1\right)\left(q^{2}-2 r+1\right),\left(q^{2}-1\right)\left(q^{2}+2 r+1\right),\left(q^{2}-1\right) 2^{f}\right\}$, where $r=2^{f}$. By [16] or [10, Remarks XI.3.12e] any subgroup of $G^{\prime} / U$ is isomorphic to some subgroup of ${ }^{2} B_{2}\left(s^{2}\right)$, when $q^{2}$ is some power of $s^{2}$, or to a subgroup of some subgroup of index $q^{4}+1, q^{4}\left(q^{4}+1\right) / 2, q^{4}\left(q^{2}-1\right)\left(q^{2}-2 r+\right.$ 1) $/ 4$ or $q^{4}\left(q^{2}-1\right)\left(q^{2}+2 r+1\right) / 4$. The last three indices are proper multiples of character degrees of $G / C$. If $T$ is properly contained in some subgroup of index $q^{4}+1$, then the result also follows easily. If $T / U$ is one of these subgroups, it suffices to note that they are not abelian (they are isomorphic to the semidirect product of the additive group of the field $\operatorname{GF}\left(q^{4}\right)$ and the multiplicative group of $\mathrm{GF}\left(q^{2}\right)$ ). Hence, we may assume that $T$ is contained in some smaller Suzuki group ${ }^{2} B_{2}\left(s^{2}\right)$. If $T / U={ }^{2} B_{2}\left(s^{2}\right)$ and $s^{2} \neq 8$ then 
the Schur multiplier of $T / U$ is 1 (by [3]). Then we can find a character of $T$ lying over $\lambda$ whose degree is a multiple of the degree of the Steinberg character of $T / U$. The result follows. If $s=8$, then the Schur multiplier is elementary abelian of order 4 . One can check in the Atlas that there is also a character of $T$ lying over $\lambda$ whose degree is a multiple of 64 . If $T$ is not a Suzuki group, it suffices to consider again the indices of the other maximal subgroups of Suzuki groups to conclude the result.

Next, we assume that $G / C={ }^{2} B_{2}(8) .3$. Then $G^{\prime} / U={ }^{2} B_{2}(8)$ and we have

$$
\operatorname{cd}(G / C)=\{1,14,105,64,195,91\} .
$$

Let $\mu \in \operatorname{Irr}(C)$ lying over $\lambda$. Let $Q$ be the inertia group of $\mu$ in $G^{\prime} C$. The indices of the maximal subgroups of $G^{\prime} C$ that contain $C$ are 2080, 1456, 560 and 65. If $Q$ is contained in some subgroup of $G^{\prime} C$ of index 560, then the degrees of the characters of $G^{\prime} C$ lying over $\mu$ are proper multiples of 14 . If $Q$ is contained in some subgroup of $G^{\prime} C$ of index 560, then the degrees of the characters of $G^{\prime} C$ lying over $\lambda$ are proper multiples of 91 . Assume now that $Q$ is contained in some subgroup of $G^{\prime} C$ of index $2080=2^{5} \cdot 5 \cdot 13$. The subgroups of $G^{\prime} C / C$ of this index are isomorphic to the dihedral group of order 14. It is clear that we can find some character of $G^{\prime} C$ lying over $\mu$ whose degree is a multiple of either $2 \cdot 2080$ or $7 \cdot 2080$. Both of these numbers are proper multiples of some character degree of $G / C$. The result follows in this case. Finally, we may assume that $Q$ is contained in some subgroup of $G^{\prime} C$ of index 65 . The result is clear if $\mu$ is not invariant under any of the elements of order 3 of $G / U$ (because the subgroups of index 65 are not abelian). Hence, we may assume that $\mu$ is invariant under some Sylow 3-subgroup of $G$. Similarly, we may assume that $\mu$ is invariant under some Sylow 7-subgroup of $G$. Hence, the inertia group $I$ of $\mu$ in $G$ contains a Hall $\{3,7\}$-subgroup of $G / C$. We conclude that

$$
3 \cdot 7|| I / C|| 2^{6} \cdot 3 \cdot 7 .
$$

Also, we may assume that $I / C$ is not a 2'-group. One can check in the Atlas that the structure of a Hall $\{2,3,7\}$-subgroup of $G / C$ is $2^{3+3}: 7: 3$. We claim that $\mu$ extends to a Sylow 2 -subgroup of $I / C$. Let $W / C$ be the normal Sylow 2-subgroup of $I / C$. If $\mu$ does not extend to $W$, then 2 divides the degree of the characters of $W$ lying over $\mu$. Then either $2 \cdot 3$ or $2 \cdot 7$ divides the degree of some of the characters of $I$ lying over $\mu$. It follows that either $2 \cdot 3 \cdot 65$ or $2 \cdot 7 \cdot 65$ divides the degree of some irreducible character of $G$ lying over $\mu$. Both numbers are proper multiples of some character degree of $G / C$. The same argument shows that we may assume that $W / C$ is a Sylow 2-subgroup of $G / C$. Hence, $I / C=2^{3+3}: 7: 3$. Let $\gamma$ be an extension of $\mu$ to $W$. If $\gamma$ does not extend to the Sylow 7-subgroup of $I / W$, then we are done (because $7 \cdot 65$ would divide some character degree). Hence, we may assume that $\gamma$ (and hence $\mu$ ) extends to $\beta \in \operatorname{Irr}(X)$, where $|X / W|=7$. Take $\alpha \in \operatorname{Irr}(X / C)$ with $\alpha(1)=7$ and put $\delta=\alpha \beta \in \operatorname{Irr}(X)$. We have that $\delta$ lies over $\mu$ and its degree is a multiple of 7 . Thus the degree of any of the characters of $G$ lying over $\delta$ is a multiple of $7 \cdot 65$. This completes the proof in this case. 
Finally, we consider the case when $G^{\prime} / U=\mathrm{L}_{2}(q)$. Since all the necessary ideas have already appeared in the previous lemmas, we will be much more sketchy in the proof.

Lemma 3.9. Let $G$ be a nonsolvable $N D A D$-group and assume that $G^{\prime} / U=$ $\mathrm{L}_{2}(q)$ for some $q \geq 4$. Then every irreducible character of $U$ is $G^{\prime}$-invariant.

Proof. As in the previous lemmas, assume that there is some irreducible character $\lambda$ of $U$ that is not $G^{\prime}$-invariant and let $T<G^{\prime}$ be the inertia group of $\lambda$ in $G^{\prime}$.

First, assume that $G / C=\mathrm{PGL}_{2}(q)$. Then

$$
\operatorname{cd}(G / C)=\{1, q-1, q, q+1\} .
$$

The subgroups of $\mathrm{L}_{2}(q)$ are described in [8, Hauptsatz II.8.27]. If $T$ is one of the groups of type $1,2,3$ or 7 , it is easy to check that the degrees of the characters of $G^{\prime}$ lying over $\lambda$ are proper multiples of some character degree of $G / C$. The groups of type 4,5 and 6 can be handled with the same ideas we have already used for the other possibilities of $G / C$. If it is of type 8 , we can use the same argument we used with the Suzuki groups.

Finally, if $G / C=M_{10}$ then $G^{\prime} / C=\mathfrak{A}_{6}$. We have $\operatorname{cd}(G / C)=\{1,9,10,16\}$ and the maximal subgroups of $\mathfrak{A}_{6}$ have indices 6, 10 and 15 . If the inertia subgroup of $\lambda$ in $G^{\prime}$ is contained in some of the subgroups of index 10, then it is easy to see that there are characters of $G$ lying over $\lambda$ whose degree is a proper multiple of 10. So we may assume that the inertia group $I$ of $\lambda$ in $G^{\prime}$ is contained in some of the subgroups of index either 6 or 15 of $G^{\prime}$. Assume first that it is a subgroup of index 6 , and hence $I / U=\mathfrak{A}_{5}$. As before, we can find a character of $I$ lying over $\lambda$ whose degree is a multiple of 3 . Hence 18 divides the degree of some character of $G$ lying over $\lambda$. It is also easy to see that $G$ is not an NDAD-group if the inertia subgroup is a proper subgroup of any of these maximal subgroups of index 6 . Thus we may assume that it is contained in a maximal subgroup of $G^{\prime}$ of index 15. The subgroups of this index of $\mathfrak{A}_{6}$ are isomorphic to $\mathfrak{S}_{4}$ and it is easy to see that in this case $G$ is not an NDAD-group either. This completes the proof.

Now we can conclude the proof of the main result of this section.

Theorem 3.10. Let $G$ be a nonsolvable $N D A D$-group and $G^{\prime} / U$ the nonabelian chief factor of $G$. Then $U$ is central in $G^{\prime}$.

Proof. Let $G$ be a minimal counterexample. Write $G^{\prime} / U=S$. By Corollary 3.4 we know that $S=\mathrm{L}_{2}(q), \mathrm{L}_{3}(4),{ }^{2} B_{2}\left(2^{2 f+1}\right), \mathfrak{A}_{7}$ or $J_{1}$. By Lemmas $3.5-3.9$, every irreducible character of $U$ is $G^{\prime}$-invariant. Thus, by Brauer's Theorem on character tables (Theorem 6.32 of [11]), $G^{\prime}$ fixes all the conjugacy classes of $U$. In particular, $S$ is isomorphic to a subgroup of the group of outer automorphisms of $U$ that fix all the conjugacy classes of $U$. By a result of Burnside (see [8, I.4 Aufgabe 12]), we know that all the prime divisors of $|S|$ divide $|U|$. By Corollary 3.4, $U$ is solvable. Let $V$ be a minimal normal subgroup of $G$ contained in $U$. By the inductive hypothesis, $U / V$ is central in $G^{\prime} / V$. Looking at the Schur multipliers of the different possibilities for $S$ and taking into account that $|U|$ should have at least three different prime divisors (and hence $|U / V|$ has at least 2 different prime divisors), we deduce that $S$ is isomorphic to $\mathrm{L}_{2}(9), \mathrm{L}_{3}(4)$ or $\mathfrak{A}_{7}$. But the Schur 
multiplier of $\mathrm{L}_{3}(4)$ has order $2^{4} \cdot 3$, while the order of $\mathrm{L}_{3}(4)$ is a multiple of 4 different prime integers. The same happens with $\mathfrak{A}_{7}$. We deduce that $S$ is isomorphic to $\mathrm{L}_{2}(9) \cong \mathfrak{A}_{6}$. In this case, we have that $V$ is an elementary abelian 5-group. Now by Corollary 5(a) of [7], we deduce that $C_{G^{\prime}}(V)$ contains a Sylow 5-subgroup of $G^{\prime}$ (because the elements of order 5 of $G^{\prime}$ are automorphisms of $V$ that fix all the conjugacy classes of $V$ ). But it is also normal in $G^{\prime}$. We deduce that $V$ is central in $G^{\prime}$. But then $U$ is nilpotent. Since $U / V$ is a $\{2,3\}$-group and $V$ is a 5 -group, we deduce that $U$ is abelian and central in $G^{\prime}$, as desired.

Now we summarize the results obtained so far.

Corollary 3.11. Let $G$ be a nonsolvable NDAD-group. Then $G^{\prime}$ is quasisimple and $G^{\prime} / Z\left(G^{\prime}\right)=\mathrm{L}_{2}(q), \mathrm{L}_{3}(4),{ }^{2} B_{2}\left(2^{2 f+1}\right), \mathfrak{A}_{7}$ or $J_{1}$.

Proof. This follows form Corollary 3.4 and Theorem 3.10.

\section{Proof of Theorem A}

In the proof of Theorem A we will use, without further explicit mention, the following well-known result. As usual, given a normal subgroup $N$ of a group $G$, we write $\operatorname{Irr}(G \mid N)=\{\chi \in \operatorname{Irr}(G) \mid N \not \leq \operatorname{Ker} \chi\}$. Also, given $\lambda \in \operatorname{Irr}(N)$ we write $\operatorname{Irr}(G \mid \lambda)=\left\{\chi \in \operatorname{Irr}(G) \mid\left[\chi_{N}, \lambda\right] \neq 0\right\}$ and $\operatorname{cd}(G \mid \lambda)=$ $\{\chi(1) \mid \chi \in \operatorname{Irr}(G \mid \lambda)\}$. We will use the following known result.

Lemma 4.1. Let $G$ be the central product of the groups $X$ and $Y$ with respect to $Z=X \cap Y$, i.e., $G=X Y,[X, Y]=1$ and $Z=X \cap Y$ is central in $G$. Then for any $\lambda \in \operatorname{Irr}(Z)$ we have

$$
\operatorname{cd}(G \mid \lambda)=\{\varphi(1) \psi(1) \mid \varphi \in \operatorname{Irr}(X \mid \lambda), \psi \in \operatorname{Irr}(Y \mid \lambda)\} .
$$

In particular, if $X$ is abelian then $\operatorname{cd}(G)=\operatorname{cd}(Y)$.

Proof. This follows from Lemma 5.1 of [12], for instance.

We also need the following easy lemma.

Lemma 4.2. Let $G$ be a nonsolvable $N D A D$-group and $C=C_{G}\left(G^{\prime}\right)$. Then $G / C$ is an almost simple NDAD-group.

Proof. It is clear that $G / C$ is an NDAD-group, so we have to show that it is almost simple. Since $G^{\prime}$ is quasi-simple, we know that $G^{\prime} C / C$ is a nonabelian simple group. We have to prove that $C_{G / C}\left(G^{\prime} C / C\right)=1$. Let $a \in G-C$ and assume that $\left[a, G^{\prime}\right] \leq C$. Then $\left[a, G^{\prime}, G^{\prime}\right]=1$. Similarly, $\left[G^{\prime}, a, G^{\prime}\right]=1$. By the three subgroups lemma, we have $\left[G^{\prime}, G^{\prime}, a\right]=1$. But $\left[G^{\prime}, G^{\prime}\right]=G^{\prime \prime}=G^{\prime}$, since $G^{\prime}$ is perfect. We deduce that $\left[G^{\prime}, a\right]=1$ so $a \in C$. This contradiction implies that $C_{G / C}\left(G^{\prime} C / C\right)=1$, as desired.

Now we can conclude the proof of Theorem A, which we restate.

Theorem 4.3. Let $G$ be a nonsolvable NDAD-group. Write $C=C_{G}\left(G^{\prime}\right)$. Then $C=Z(G)$ and one of the following holds:

(i) $G=S \times C$, where $S$ is $J_{1}$ or ${ }^{2} B_{2}\left(2^{2 f+1}\right)$ for some $f>1$;

(ii) $G^{\prime}=\mathfrak{A}_{7}$ and $\left|G / C G^{\prime}\right| \leq 2$.

(iii) $G^{\prime}={ }^{2} B_{2}(8)$ and $\left|G / C G^{\prime}\right|$ divides 3 .

(iv) $G^{\prime}=\mathrm{L}_{3}(4)$ and $G / C=\mathrm{L}_{3}(4), \mathrm{L}_{3}(4) .2_{1}, \mathrm{~L}_{3}(4) .3$, or $\mathrm{L}_{3}(4) .6$. 
(v) $G^{\prime}=\mathrm{L}_{2}(q)$ or $\mathrm{SL}_{2}(q)$ and $G / C=\mathrm{PGL}_{2}(q)$.

(vi) $G^{\prime}$ is $\mathfrak{A}_{6}$ or the 3 -fold cover of $\mathfrak{A}_{6}$ and $G / C=M_{10}$.

Conversely, if $C=Z(G)$ and any of (i)-(vi) holds, then $G$ is a nonsolvable NDAD-group.

Proof. By Corollary 3.11 we know that $G^{\prime}$ is quasi-simple and $G^{\prime} / Z\left(G^{\prime}\right)=$ $\mathrm{L}_{2}(q), \mathrm{L}_{3}(4),{ }^{2} B_{2}\left(2^{2 f+1}\right), \mathfrak{A}_{7}$ or $J_{1}$. Observe also that $G / C$ is an almost simple NDAD-group by Lemma 4.2. Assume first that $G^{\prime} / Z\left(G^{\prime}\right)=J_{1}$ or ${ }^{2} B_{2}\left(2^{2 f+1}\right)$ for some $f>1$. Since these groups have trivial Schur multiplier, we know that $Z\left(G^{\prime}\right)=1$. We have that $C \times G^{\prime} \leq G$. Since $G / C$ is an almost simple NDAD-group, it follows from Theorem 2.8 that $G / C=J_{1}$ or ${ }^{2} B_{2}\left(2^{2 f+1}\right)$. Thus (i) holds.

Next, assume that $G^{\prime} / Z\left(G^{\prime}\right)=\mathfrak{A}_{7}$. By Theorem 2.8 , we know that $G / C=$ $\mathfrak{A}_{7}$ or $\mathfrak{S}_{7}$ In both cases, we have that $6 \in \operatorname{cd}(G / C)$. Assume now that $Z\left(G^{\prime}\right)>1$. One can check in the Atlas that 24 or $36 \in \operatorname{cd}\left(G^{\prime}\right)$. If we choose $\chi \in \operatorname{Irr}(G)$ lying over some irreducible character of $G^{\prime}$ of degree 24 or 36 , we see that 6 is a proper divisor of $\chi(1)$. It follows that $G$ is not an NDAD-group. Hence $Z\left(G^{\prime}\right)=1$. We deduce that (ii) holds.

Now, assume that $G^{\prime} / Z\left(G^{\prime}\right)={ }^{2} B_{2}(8)$. By Theorem 2.8, we know that $G / C={ }^{2} B_{2}(8)$ or ${ }^{2} B_{2}(8) .3$. In both cases, we have that $14 \in \operatorname{cd}(G / C)$. Assume now that $Z\left(G^{\prime}\right)>1$. One can check in the Atlas that $56 \in \operatorname{cd}\left(G^{\prime}\right)$. If we choose $\chi \in \operatorname{Irr}(G)$ lying over some irreducible character of $G^{\prime}$ of degree 56 , we see that 14 is a proper divisor of $\chi(1)$. It follows that $G$ is not an NDAD-group. Hence $Z\left(G^{\prime}\right)=1$. We deduce that (iii) holds.

Now, assume that $G^{\prime} / Z\left(G^{\prime}\right)=\mathrm{L}_{3}(4)$. By Theorem 2.8, we know that $G / C=\mathrm{L}_{3}(4), \mathrm{L}_{3}(4) .2_{1}, \mathrm{~L}_{3}(4) .3$, or $\mathrm{L}_{3}(4) .6$. The subgroup $C G^{\prime}$ is the central product of $C$ and $G^{\prime}$ with respect to $Z\left(G^{\prime}\right)$.

If 3 divides $\left|Z\left(G^{\prime}\right)\right|$ we can check in the Atlas that 21 and 84 belong to $\operatorname{cd}\left(G^{\prime}\right)$. We have that for any $\gamma \in \operatorname{Irr}(C)$ lying over a linear character of $Z\left(G^{\prime}\right)$ of order $3,21 \gamma(1)$ and $84 \gamma(1) \in \operatorname{cd}\left(C G^{\prime}\right)$. Since $G / C G^{\prime}$ is a cyclic group whose order divides 6 and the characters of $G^{\prime}$ of degree 21 and 84 are $G$-invariant, it follows that $G$ is not an NDAD-group.

Next, assume that 4 divides the exponent of $Z\left(G^{\prime}\right)$. Then we can check in the Atlas that $80 \in \operatorname{cd}\left(G^{\prime}\right)$, so there is some multiple of 80 in $\operatorname{cd}(G)$. On the other hand, $20 \in \operatorname{cd}(G / C)$. Thus $G$ is not an NDAD-group.

Assume now that the exponent of $Z\left(G^{\prime}\right)$ is 2 . We can see in the Atlas that in this case

$$
\operatorname{cd}\left(G^{\prime}\right)=\operatorname{cd}\left(G^{\prime} / Z\left(G^{\prime}\right)\right) \cup\{10,28,36,70,90\} .
$$

We claim that $C$ is abelian. Otherwise, $C$ has an irreducible character of 2-power degree lying over a nonprincipal character of $Z\left(G^{\prime}\right)$. It follows that $2 \cdot 90$ divides the degree of some irreducible character of $C G^{\prime}$ and hence of some irreducible character of $G$. But $20 \in \operatorname{cd}(G / C)$ so it follows that $G$ is not an NDAD-group. This contradiction proves our claim. In particular, $\operatorname{cd}\left(C G^{\prime}\right)=\operatorname{cd}\left(G^{\prime}\right)$. Next, we claim that $C$ is central in $G$. Assume not. Clearly, $C$ is central in $C G^{\prime}$ and $C / Z\left(G^{\prime}\right)$ is central in $G / Z\left(G^{\prime}\right)$. Then there exists some nonprincipal character of $\operatorname{Irr}\left(C \mid Z\left(G^{\prime}\right)\right)$ whose inertia group is a proper subgroup of $G$ that contains $C G^{\prime}$. Since $|G / C G|$ divides 6 , we deduce that either a multiple of $2 \cdot 90$ or a multiple of $3 \cdot 90$ belongs to $\operatorname{cd}(G)$. But 
20 and either 45 or 90 belong to $\operatorname{cd}(G / C)$. In any case, we deduce that $G$ is not an NDAD-group. This is again a contradiction. We conclude that $C=Z(G)$

Next, we consider the case when $Z\left(G^{\prime}\right)=2 \times 2$. Assume that 3 divides $\left|G / C G^{\prime}\right|$. Then there is an element of order 3 of $G / C G^{\prime}$ that permutes transitively the 3 subgroups of order 2 of $Z\left(G^{\prime}\right)$. This implies that a multiple of 270 belongs to $\operatorname{cd}(G)$. But $G / C$ has some irreducible character of degree 90 or 45 . This contradiction implies that $|G / C G|$ divides 2 . If $Z\left(G^{\prime}\right)$ is cyclic of order 2 , then one can see in the Atlas that $\left|G / C G^{\prime}\right|$ also divides 2. Since $C G^{\prime}$ is not an NDAD-group, we deduce that $\left|G / C G^{\prime}\right|=2$. But then one can check in the Atlas that 35 and 70 belong to $\operatorname{cd}(G)$. Thus $G$ is not an NDAD-group either.

Hence $Z\left(G^{\prime}\right)=1$ and it is easy to see that (iv) holds.

Finally, we may assume that $G^{\prime} / Z\left(G^{\prime}\right)=\mathrm{L}_{2}(q)$. Then $G / C=\operatorname{PGL}_{2}(q)$ or $M_{10}$. Assume that $Z\left(G^{\prime}\right)=1$. Then clearly $C=Z(G)$ and (v) or (vi) holds. Hence, we may assume that $Z\left(G^{\prime}\right)>1$. In particular, we may assume that $q \geq 5$ is odd. First, we consider the case $q \neq 9$. Then $\left|Z\left(G^{\prime}\right)\right|=2$. Arguing as in the case of $\mathrm{L}_{3}(4)$ one can see that $C=Z(G)$ and (v) holds.

Thus we may assume that $q=9$. Assume first that $G / C=\mathrm{PGL}_{2}(9)$. Then $\operatorname{cd}(G / C)=\{1,8,9,10\}$. If 3 divides $\left|Z\left(G^{\prime}\right)\right|$, then 3 and 9 belong to $\operatorname{cd}\left(G^{\prime}\right)$. Hence, it is not difficult to see that $G$ is not an NDAD-group. Thus, we may assume that $\left|Z\left(G^{\prime}\right)\right|=2$. Now one can conclude that $C=Z(G)$ and (v) holds.

Finally, we assume that $G / C=M_{10}$. Then $\operatorname{cd}(G / C)=\{1,9,10,16\}$. Using the information in the Atlas one can see that in this case if 2 divides $\left|Z\left(G^{\prime}\right)\right|$ then $G$ is not an NDAD-group. Thus we may assume that $\left|Z\left(G^{\prime}\right)\right|=$ 3 or $\left|Z\left(G^{\prime}\right)\right|=1$. We know that $G^{\prime}$ has an irreducible character of degree 15 . If $C$ is not central in $G$, then we can find some irreducible character of $G$ of degree a multiple of 30. But this implies that $G$ is not an NDAD-group. We conclude that $C=Z(G)$ and (vi) holds.

Now we prove that if $C=Z(G)$ and any of (i)-(vi) holds, then $G$ is an NDAD-group. In fact, it is easy to determine explicitly the sets of character degrees of these groups. It suffices to observe that in any of the cases $\operatorname{cd}(G)=\operatorname{cd}(G / C)$, so the set of character degrees of a nonsolvable NDAD-group coincides with the set of character degrees of an almost simple NDAD-groups. More precisely, if (i) holds then

$$
\operatorname{cd}(G)=\{1,56,76,77,120,133,209\}
$$

or

$\operatorname{cd}(G)=\left\{1, q^{4}, q^{4}+1,\left(q^{2}-1\right)\left(q^{2}-2 r+1\right),\left(q^{2}-1\right)\left(q^{2}+2 r+1\right),\left(q^{2}-1\right) \cdot 2^{f}\right\}$, where $q^{2}=2^{2 f+1}$ and $r=2^{f}$. If (ii) holds, then

$$
\operatorname{cd}(G)=\{1,6,10,14,15,21,35\}
$$

or

$$
\operatorname{cd}(G)=\{1,6,14,15,20,21,35\}
$$

If (iii) holds, then

$$
\operatorname{cd}(G)=\{1,14,35,64,65,91\}
$$


or

$$
\operatorname{cd}(G)=\{1,14,64,91,105,195\} .
$$

If (iv) holds, then $\operatorname{cd}(G)=\operatorname{cd}(G / C)$ and one can see in the Atlas that these are:

$$
\begin{gathered}
\operatorname{cd}\left(\mathrm{L}_{3}(4)\right)=\{1,20,35,45,63,64\}, \\
\operatorname{cd}\left(\mathrm{L}_{3}(4) .2_{1}\right)=\{1,20,35,90,126,64\}, \\
\operatorname{cd}\left(\mathrm{L}_{3}(4) .3\right)=\{1,20,105,45,63,64\}
\end{gathered}
$$

or

$$
\operatorname{cd}\left(\mathrm{L}_{3}(4) .6\right)=\{1,20,105,90,126,64\} .
$$

If (v) holds, then

$$
\operatorname{cd}(G)=\{1, q-1, q, q+1\}
$$

with $q=p^{f} \geq 4$ a prime power. If (vi) holds, then

$$
\operatorname{cd}(G)=\{1,9,10,16\}
$$

or

$$
\operatorname{cd}(G)=\{1,6,9,10,15,16\} .
$$

\section{Proof of Corollaries B and C}

The following corollary solves Conjecture A of [13].

Corollary 5.1. Let $G$ be a finite NDAD-group. Then $|\operatorname{cd}(G)| \leq 7$.

Proof. It was proved in Corollary $\mathrm{C}$ of [13] that if $G$ is a solvable NDADgroup, then $|\operatorname{cd}(G)| \leq 4$. In the proof of Theorem A, we have explicitly determined the possible sets of character degrees of the nonsolvable NDADgroups. One can easily see that in any case $|\operatorname{cd}(G)| \leq 7$.

Finally, we prove Corollary C.

Corollary 5.2 (Huppert). If $S=\mathrm{L}_{2}\left(2^{f}\right)$ for some $f>1,{ }^{2} B_{2}\left(2^{2 f+1}\right)$ for some $f \geq 1, \mathrm{~L}_{3}(4), J_{1}$ or $\mathfrak{A}_{7}$ and $G$ is a finite group with $\operatorname{cd}(G)=\operatorname{cd}(S)$, then $G=S \times A$ for some abelian group $A$.

Proof. Observe that all these simple groups are NDAD-groups. Assume first that for some nonsolvable group $\operatorname{cd}(G)=\operatorname{cd}(S)$ with $\operatorname{cd}(G)=\operatorname{cd}(S)$. Hence, $G$ is one of the groups that appear in the statement of Theorem A. The result follows from the determination of the sets of character degrees of the nonsolvable NDAD-groups given at the end of the proof of Theorem A.

Now, assume that $\operatorname{cd}(G)=\operatorname{cd}(S)$ for some solvable group $G$. By Theorem B of [13], we know that $|\operatorname{cd}(G)| \leq 3$ or $|\operatorname{cd}(G)|=4$ and $\operatorname{cd}(G)$ contains at least two multiples of some prime number $p$. Since for any of our simple groups (and in fact for any simple group) $|\operatorname{cd}(G)| \geq 4$ and if $|\operatorname{cd}(G)|=4$ then $\operatorname{cd}(G)$ is a set of pairwise coprime integers, we obtain a contradiction. This completes the proof of the corollary. 


\section{REFERENCES}

[1] YA. G. Berkovich, E. M. Zhmud', Characters of Finite Groups, part 2. (Translations of Mathematical Monographs 181, 1999).

[2] R.W. Carter, Finite groups of Lie type. Wiley-Interscience, New York, 1985.

[3] J.H. Conway, R.T. Curtis, S.P. Norton, R.A. Parker, R.A. Wilson, Atlas of Finite Groups. Clarendon Press, Oxford, 1985.

[4] F. Digne, J. Michel, Groupes réductifs non connexes. Ann. Sci. École Norm. Sup. 27 (1994), 345-406.

[5] M. GeCK, G. Pfeiffer, Characters of finite Coxeter groups and Iwahori-Hecke algebras. Oxford University Press, Oxford, 2000.

[6] D. Gorenstein, R. Lyons, R. Solomon, The classification of the finite simple groups, Number 3. Mathematical Surveys and Monographs, Amer. Math. Soc., Providence, 1994.

[7] M. Hertweck, Class-preserving automorphisms of finite groups. J. Algebra 241 (2001), no. 1, 1-26.

[8] B. Huppert, Endliche Gruppen I. Springer-Verlag, Berlin, 1967.

[9] B. Huppert, Some simple groups which are determined by the set of their character degrees I. Illinois J. Math. 44 (2000), 828-842.

[10] B. Huppert, N. Blackburn, Finite Groups III. Springer-Verlag, New York, 1982.

[11] I. M. IsAacs, Character Theory of Finite Groups. Dover, New York, 1994.

[12] I. M. IsaAcs, G. Malle, G. Navarro, A reduction theorem for the McKay conjecture, submitted.

[13] M. Lewis, A. Moretó, T. Wolf, Non-divisibility among character degrees. J. Group Theory 8 (2005), 561-588.

[14] G. LuszTig, On the representations of reductive groups with disconnected centre. Pp. 157-166 in: Orbites unipotentes et représentations, I. Astérisque 168, (1988).

[15] A. Moretó, Complex group algebras of finite groups: Brauer's Problem 1, to appear in Adv. Math.

[16] M. Suzuki, On a class of doubly transitive groups, Ann. Math. 75 (1962), 104-145.

[17] H. N. WARD, On Ree's series of simple groups. Trans. Amer. Math. Soc. 121 (1966), 62-89.

G.M.: Fachbereich Mathematik, Universität Kaiserslautern, Postfach 3049,

D-67653 Kaiserslautern, Germany.

E-mail address: malle@mathematik.uni-kl.de

A.M.: Departament D'Àlgebra, Universitat de València 46100 Burjassot. ValÈnCia. Spain

E-mail address: Alexander.Moreto@uv.es 